\section{Neue Chance bei Hodgkin-Lymphomen}

Brentuximab Vedotin (Adcetris ${ }^{\circledast}$ ) hat am 19. Juli 2012 vom Ausschuss für Humanarzneimittel der Europäischen MedizinAgentur die Empfehlung für die Zulassung bei rezidiviertem oder refraktärem Hodgkin-Lymphom (HL) sowie systemischem anaplastisch-großzelligem Lymphom (sALCL) erhalten. In zwei PhaseII-Studien erzielte Brentuximab Vedotin hohe objektive Ansprechraten (86\% bei sALCL, 75\% bei HL) mit vollständigen Remissionen bei 59\% der Patienten mit sALCL und $33 \%$ der Patienten mit HL. Gegenüber konventioneller Chemotherapie war dabei die Nebenwirkungsrate geringer. Die häufigsten unerwünschten Ereignisse (> 20\%) waren Neutropenie, periphere sensorische Neuropathie, Fatigue, Übelkeit, Anämie, Infektionen der oberen Atemwege, Diarrhö, Fieber, Ausschlag, Thrombozytopenie, Husten und Erbrechen.

Nach Information von Takeda

\section{$\mathrm{NHL}$ : Erhaltungstherapie überlegen}

Neue Studiendaten bestätigen die Rolle von Rituximab (MabThera ${ }^{\oplus}$ ) zur Therapie des follikulären Non-HodgkinLymphoms (NHL). In einer Phase-IIIStudie mit 514 Patienten mit follikulären, Marginalzonen-, Mantelzell-, kleinen lymphozytischen Lymphomen und Waldenström-Makroglobulinämie war Rituximab plus Bendamustin kombiniert dem bisherigen Standard R-CHOP bezüglich des progressionsfreien Überlebens mit im Median 69,5 vs. 31,2 Monaten signifikant überlegen ( $\mathrm{p}=$ 0,0000148 ) [Rummel MJ et al. J Clin Oncol. 2012;30(suppl):Abstr 3]. Die National LymphoCare Study (NLCS) belegte die Wirksamkeit einer Rituximab-Erhaltungstherapie [Nastoupil L et al. Haematologica. 2012; 97(S1):95-5(Abstr 238)]. 53 Monate nach Induktionsbeginn lag das Risiko für Progression oder Tod bei Erhaltungstherapie um 31\% niedriger ( $p=0,003$ ), die Zeit bis zur nächsten Lymphom-Therapie war um 27 \% länger als im Vergleichsarm ( $\mathrm{p}=$ $0,03)$.

Nach Informationen von Roche

Kolorektales Karzinom

\title{
Angiogenesehemmung über Progression hinaus
}

Patienten mit metastasiertem kolorektalem Karzinom überleben länger, wenn die Angiogenesehemmung über die Progression hinaus fortgeführt und mit der Zweitlinientherapie kombiniert wird.

Üblicherweise wird mit dem Angiogenesehemmer Bevacizumab (Avastin ${ }^{\circ}$ ) bis zur Tumorprogression behandelt. Die darüber hinaus fortgesetzte Gabe kann jedoch das Gesamtüberleben der Patienten verbessern, so das Ergebnis einer PhaseIII-Studie, das Dirk Arnold, Hamburg, vorstellte. In der internationalen multizentrischen Studie (ML18147) waren 820 Patienten mit metastasiertem kolorektalem Karzinom, die in der Erstlinie eine Oxaliplatin- oder Irinotecan-basierte Chemotherapie und zusätzlich Bevacizumab erhalten hatten, bei Tumorprogression mit einer anderen Zweitlinien-Chemotherapie behandelt worden. Eine Patientengruppe erhielt bis zur erneuten Krankheitsprogression zusätzlich weiterhin den Angiogenesehemmer. Es zeigte sich laut Arnold ein signifikanter Vorteil der Patienten unter fortgesetzter Gabe des Antikörpers: In der Bevacizumab-Gruppe wurde das Gesamtüberleben von durchschnittlich 9,8 auf 11,2 Monate und damit signifikant um 1,4 Monate verlängert $(p=0,0062)$. Dieser Überlebensvorteil war unabhängig vom Alter der Patienten und weitgehend unabhängig vom Allgemeinzustand, der Zahl der befallenen Organe und der Art der Erstlinienbehandlung. Die gezielte Hemmung des Wachstumsfaktors VEGF über die Progression hält Arnold für folgerichtig: „Schließlich ist der Wachstumsfaktor VEGF der stärkste Treiber der Tumorangiogenese“.

Christine Vetter

Pressekonferenz "Aktuelles vom amerikanischen Krebskongress 2012" am 14. Juni 2012 in Frankfurt/Main; Veranstalter: Roche.

\section{Nach Chemo wirksam weiterbehandeln}

\section{Durch die Zulassung von neuen Wirkstoffen und deren sequenziel- len Einsatz hat sich die Prognose beim metastasierten kastrationsre- sistenten Prostatakarzinom (mCRPC) in den letzten Jahren erheblich verbessert.}

Eine aktuelle Auswertung der Zulassungsstudie von Abirateronacetat (Zytiga ${ }^{\circledR}$ ) in Kombination mit Prednison/Prednisolon (AAP) zeigt, dass sich durch den sequenziellen Einsatz von Docetaxel firstline sowie Abirateronacetat mit Prednison/Prednisolon secondline das mediane Überleben der Patienten, gemessen ab der ersten Docetaxel-Gabe (DP), auf im Median 32,6 Monate verlängerte [Goodman $\mathrm{OB}$ et al. J Clin Oncol. 2012;30(Suppl):Abstr 4558]. Abirateronacetat $(\mathrm{n}=797)$ hatte in der Studie COU-AA-301 bei mCRPC-Patienten nach Versagen von ein bis zwei Che-
motherapie-Regimen inklusive Docetaxel das mediane Überleben gegenüber Placebo $(\mathrm{n}=398)$ (je plus Prednison/Prednisolon: AAP vs. PP) signifikant um 4,6 Monate verlängert (15,8 vs. 11,2 Monate, Hazard Ratio $0,74, p<0,0001)$. Der Überlebensvorteil war dabei unabhängig vom Grund des Abbruchs der Docetaxel-Therapie und unabhängig von der Zeitspanne zwischen letzter Docetaxel- und erster Abirateronacetat-Dosis. Eine Ausnahme bildeten Patienten, deren Docetaxel-Therapie 3 Monate oder weniger gedauert hatte hier ließ sich nur ein Trend erkennen. Die Daten belegen, dass Patienten mit mCRPC durch eine Therapie bestehend aus Docetaxel in der Erstlinie und Abirateronacetat mit Prednison/Prednisolon secondline die Chance haben, im Median noch weitere knapp drei Jahre zu überleben. red

Nach Information von Janssen-Cilag 